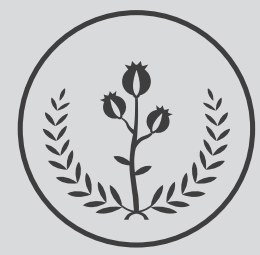

FUCS
$=1$

№3. 2022

\title{
Conjuntivitis leñosa
}

\section{Ligneous conjunctivitis}

Claudia Lucía Téllez MD

Omar Toncel $\mathbf{M D}^{\mathrm{b}}$

Alejandro Arias-Gómez MD

${ }^{a}$ Oculoplástica, Órbita y Vías Lagrimales Hospital de San José, Profesor Asistente Fundación Universitaria de Ciencias de la Salud,

Bogotá DC, Colombia.

${ }^{b}$ Oftalmología. Fundación Universitaria de Ciencias de la Salud, Bogotá DC, Colombia.

\section{R E S U M EN}

Objetivo: presentar y describir el cuadro clínico de un paciente con conjuntivitis leñosa en el Hospital de San José de Bogotá. Diseño: estudio descriptivo, retrospectivo y reporte de caso. Metodología: revisión y análisis de la literatura con referencia al diagnóstico de la patología, con variables en lo posible similares a las encontradas en el paciente, con el propósito de caracterizar la condición y determinar el manejo satisfactorio. Presentación del caso: paciente de 19 años con manifestación temprana (desde el nacimiento) consistente en aparición de lesiones tipo papila, de tamaño gigante, en conjuntiva tarsal superior en ambos ojos. Al examen oftalmológico se evidenciaron papilas confluentes con masas lobuladas dependientes de la conjuntiva tarsal superior. La biopsia confirmó el diagnóstico de conjuntivitis leñosa congénita.

Palabras clave: conjuntivitis leñosa, queratitis, déficit de plasminógeno.

(C) 2022 Fundación Universitaria de Ciencias de la Salud - FUCS. Este es un artículo Open Access bajo la licencia CC BY-NC-ND (http:// creativecommons.org/licenses/by-nc-nd/4.0/)

\section{INFORMACIÓN DEL ARTÍCULO}

Historia del artículo:

Fecha recibido: agosto 5 de 2019 Fecha aceptado: mayo 21 de 2020
Autor para correspondencia. Dra. Claudia Lucía Téllez: claudiatellexm@yahoo.co
DOI

10.31260/RepertMedCir.01217372.984 
Objective: to present and describe the clinical manifestations of ligneous (woody) conjunctivitis in a patient seen at Hospital de San José in Bogotá. Design: descriptive, retrospective study and case report. Methodology: review and analysis of the literature with reference to the diagnosis of the pathology, with variables as similar as possible to those found in the patient, with the purpose of characterizing the condition and determining a satisfactory management. Case presentation: a 19-year-old patient with early manifestations (from birth) consisting of giant papilla type lesions in upper tarsal conjunctiva in both eyes. Confluent papillae with lobed masses dependent on the superior tarsal conjunctiva were evidenced upon ophthalmological exam. The biopsy confirmed the diagnosis of congenital woody conjunctivitis.

Key words: Woody conjunctivitis, keratitis, plasminogen deficiency.

(C) 2022 Fundación Universitaria de Ciencias de la Salud - FUCS, This is an open access article under the CC BY-NC-ND license (http:// creativecommons.org/licenses/by-nc-nd/4.0/)

\section{INTRODUCCIÓN}

La conjuntivitis leñosa es una forma crónica rara caracterizada por el desarrollo de tejido pseudomembranoso rico en fibrina con apariencia de madera en la conjuntiva tarsal $^{1}$, que puede afectar eventualmente la agudeza visual ${ }^{2}$ debido al compromiso corneal en cerca de 20 a 30\% de los casos.

La mayoría de los afectados son niños e infantes, sin embargo puede manifestarse a cualquier edad. Ambos ojos están comprometidos en cerca de $51 \%$ de los casos. Recientemente ha sido asociada con otros trastornos de baja incidencia como la hipoplasminogenemia, siendo en $80 \%$ de los casos su primera manifestación. Así mismo se encuentra asociada con irritantes externos y cirugía ocular. No hay consenso en la literatura sobre el manejo óptimo de los pacientes con esta condición debido a su baja incidencia. El diagnóstico y manejo temprano puede prevenir el empeoramiento de la visión como resultado del compromiso corneano, por esta razón los pacientes deben ser manejados en forma interdisciplinaria, con un seguimiento regular y apropiado.

Al examen oftalmológico, en la biomicroscopía los principales hallazgos revelan edema de ambos párpados, lesiones tipo pseudomembranas blanquecinas o membranas sésiles gruesas, firmemente adheridas a la conjuntiva tarsal superior e inferior, con escaso compromiso de la bulbar y cicatrices en la tarsal.,

Consideramos importante reportar nuestro caso ya que se trata de una presentación típica de una patología de baja frecuencia, poco documentada en la literatura médica y en nuestro medio. Identificar estos hallazgos favorece el diagnóstico y tratamientos oportunos de la entidad que puede generar un importante compromiso ocular por afectación corneal y visual secundarias, así como repercusión sistémica derivada de la alteración en el sistema hematopoyético por su asociación en $80 \%$ de los casos con hipoplasminogenemia.

\section{CASO CLÍNICO}

Paciente masculino de 19 años que consulta por cuadro clínico crónico que tuvo inicio al nacimiento, caracterizado por aparición en la conjuntiva tarsal de ambos ojos de lesiones tipo papila, de tamaño gigante, que al parecer ha recibido manejo quirúrgico (excisión de lesiones) en múltiples ocasiones de forma extrainstitucional. En el momento del ingreso manifestó recidiva y reaparición de las mismas (no trae reporte de patología ni copia de la historia clínica), anotando que utiliza manejo tópico con ácido N-Acetil aspartil glutámico, hialuronato de sodio y ciclosporina, aunque no es clara la fecha de inicio del tratamiento.

En los antecedentes se encuentra una cirugía de extracción de catarata en ojo derecho con implante de lente intraocular realizada hace 8 años en otra institución oftalmológica, deficiencia de plasminógeno, hidrocefalia que requirió implante de válvula ventriculoperitoneal, retardo mental leve a moderado y un síndrome convulsivo secundario para lo cual recibe tratamiento con levetiracetam y propanolol clorhidrato. Cabe destacar que el paciente y la madre no relatan antecedentes familiares de importancia.

Al examen oftalmológico se encontró la agudeza visual del ojo derecho en 20/400, y el ojo izquierdo 20/800. En la biomicroscopía se evidenciaron en ambos ojos múltiples lesiones tipo papila, confluentes que formaban una masa multilobulada dependiente de la conjuntiva tarsal superior (figuras 1, 2A y B). 


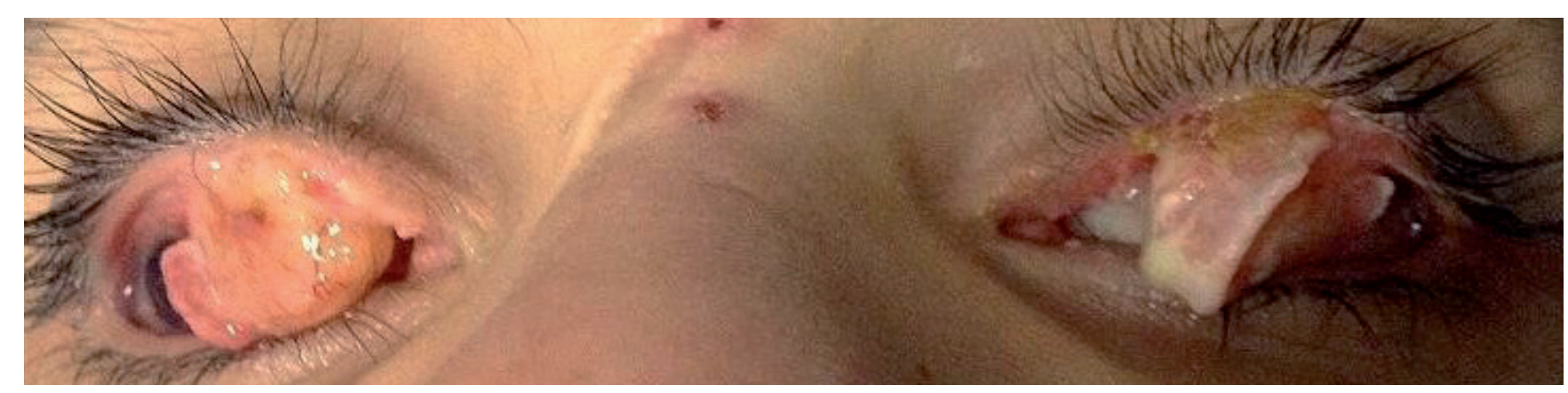

Figura 1. Lesiones en conjuntiva tarsal superior con eversión del párpado, produciendo alteración permanente de la superficie ocular. Fuente: los autores.
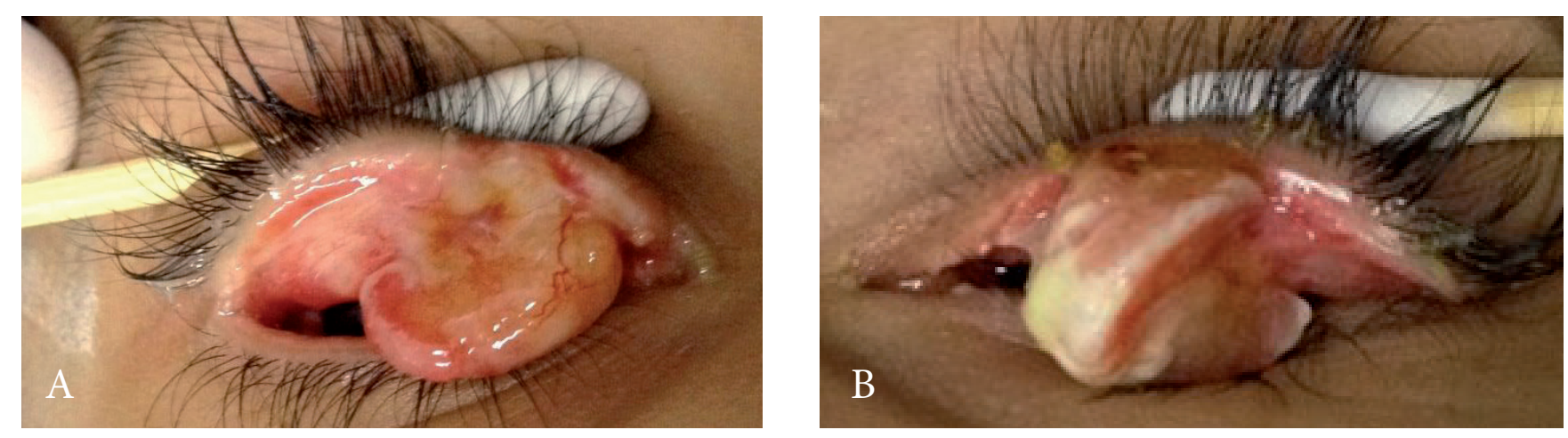

Figura 2. Acercamiento del aspecto leñoso de la lesión conjuntival tarsal en ojos derecho (A) e izquierdo (B). Fuente: los autores.

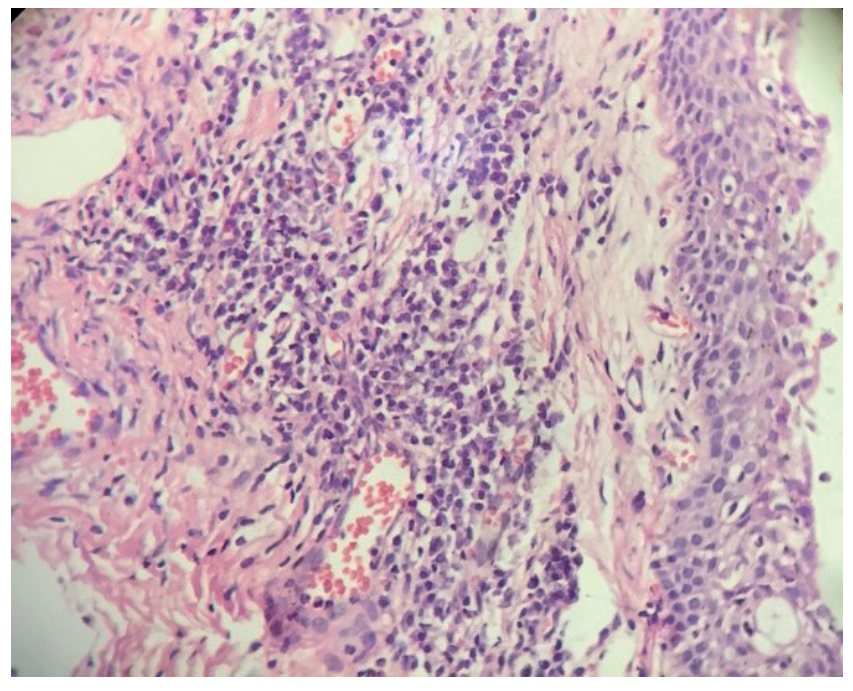

Figura 3. Corte histológico de conjuntiva con infiltrado inflamatorio crónico de linfocitos y plasmocitos. Fuente: los autores.

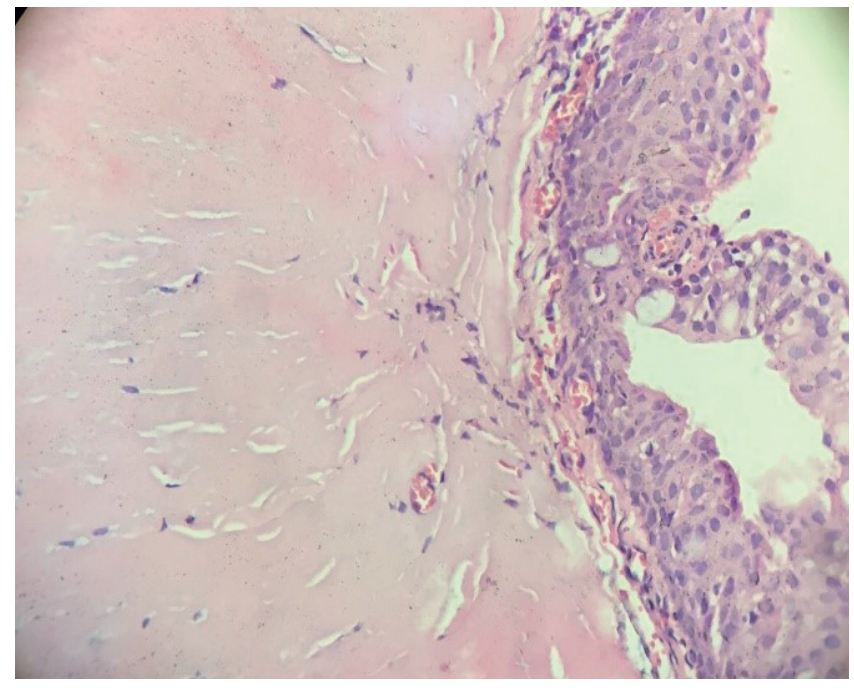

Figura 4. Material amorfo denso. Fuente: los autores. 
En el ojo derecho se apreció queratitis punteada superficial difusa leve y un lente intraocular en posición. En el izquierdo la córnea mostró vascularización superficial y profunda, y un leucoma paracentral inferonasal con opacidad central en el cristalino. La presión intraocular y el fondo de ojo fueron normales en ambos ojos. Dados los hallazgos en la biomicroscopía se diagnosticó probable conjuntivitis leñosa y se ordenó la resección quirúrgica de las lesiones conjuntivales con avanzamiento conjuntival en ambos ojos bajo anestesia general; así mismo se solicitó el estudio histopatológico de las lesiones y la valoración por el servicio de hematología para el manejo integral del paciente.
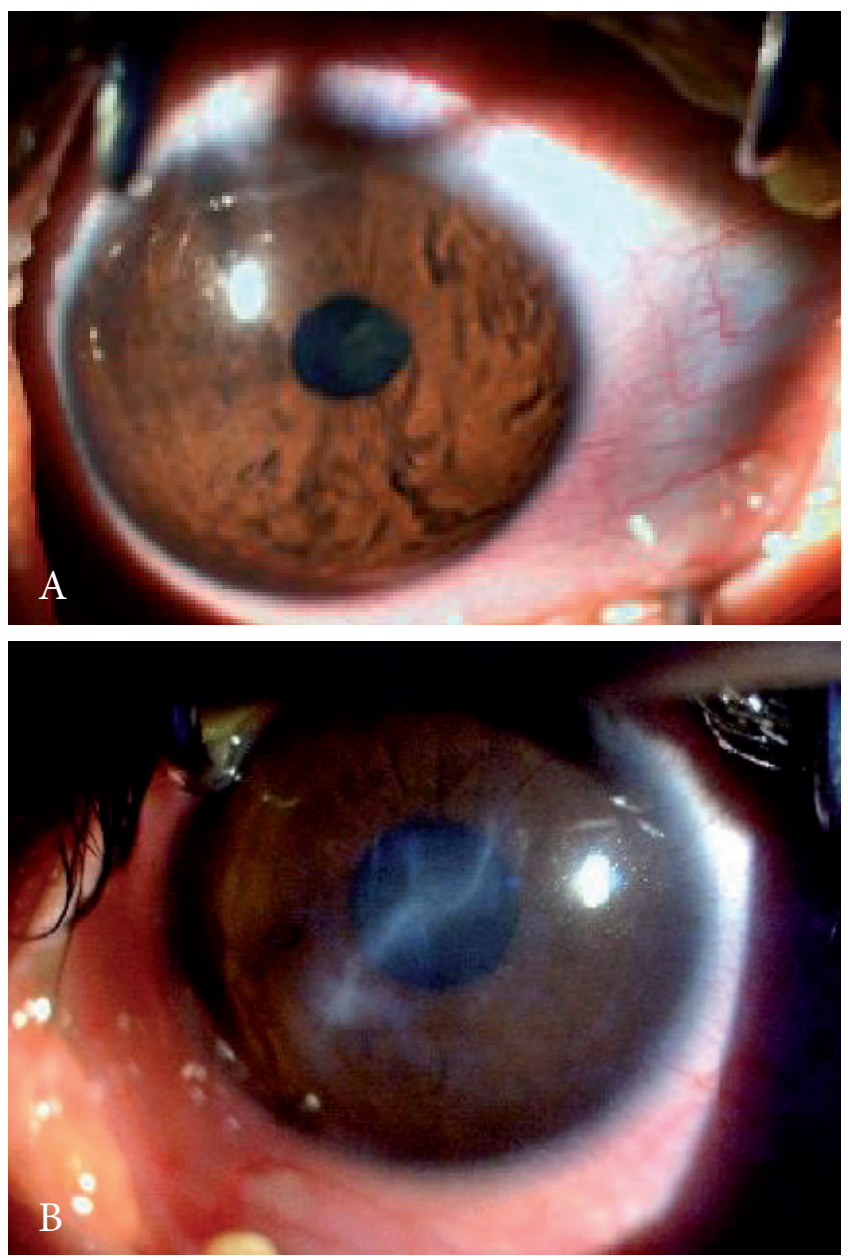

Figura 5 A. Biomicroscopía del ojo derecho con escasas opacidades superiores en córnea. Figura 5 B. En el ojo izquierdo se aprecia leucoma central. Fuente: los autores.

Después de la intervención se inició tratamiento tópico con ciprofloxacina cada 4 horas en ambos ojos, enoxaparina subcutánea $40 \mathrm{mg}$ al día, propanolol $20 \mathrm{mg}$ al día vía oral y levotiracetam $1 \mathrm{~g}$ cada 8 horas. La evolución fue satisfactoria y se dió de alta al día siguiente, con controles por consulta externa.

La histopatología reportó un material amorfo acelular rodeado por epitelio conjuntival, con denso infiltrado inflamatorio agudo y crónico, confirmando la sospecha diagnóstica de conjuntivitis leñosa en ambos ojos (figuras 3 y 4). Luego de 1 mes de haber realizado el procedimiento quirúrgico el paciente no mostró signos de recidiva. En el posoperatorio luego de 1 mes de la cirugía se vieron opacidades en la córnea del ojo derecho y leucoma central en el ojo izquierdo (figuras 5 A y B).

\section{IS C USIÓN}

La conjuntivitis leñosa es un raro desorden hereditario de carácter autosómico recesivo siendo la causa más frecuente la mutación del gen del plasminógeno K19E. ${ }^{5}$

Aunque esta condición fue reportada en 1847 y su descripción histológica detallada publicada en 1924, el término "conjuntivitis leñosa" fue introducido por Borel en 1933 y su relación con la deficiencia de plasminógeno fue establecida en $1997 . .^{6-8}$ Se caracteriza por la presencia de lesiones membranosas o pseudomembranosas en la superficie de la conjuntiva tarsal. ${ }^{9}$ En la mayoría de los pacientes se detecta un déficit de plasminógeno, que media el proceso de fibrinólisis extracelular requerido en el proceso normal de cicatrización para remover la matriz rica en fibrina y llevar a cabo el remodelamiento del tejido de granulación. ${ }^{10}$ Si bien el compromiso ocular es el más frecuente afectando alrededor de $80 \%$ de los individuos que la padecen, cursa con una gran variedad de manifestaciones sistémicas incluyendo periodontitis, cálculos renales, vulvovaginitis, enfermedad respiratoria, otitis media, lesiones dermatológicas e hidrocefalia, entre otras. ${ }^{11}$

En el paciente existe la asociación con hidrocefalia, documentada previamente. Algunos autores describen la relación de esta entidad con el uso de irritantes químicos $\mathrm{y}$ con algunos procedimientos quirúrgicos. ${ }^{11}$ En nuestra población no hay datos exactos sobre la incidencia y prevalencia de esta afección. Los registros europeos muestran una prevalencia de 1.6 en 1 millon. ${ }^{1}$ En la histopatología se observa material amorfo, eosinofílico subepitelial positivo con tinción PAS y negativo rojo Congo con células de respuesta inflamatoria crónica. ${ }^{12-14}$ Se ha intentado la terapia tópica con corticoesteroides y hialuronidasa. ${ }^{2}$ Respecto a la ciclosporina A, está indicada en los pacientes con respuesta refractaria a otros tipos de tratamiento. Este fármaco es un inmunomodulador altamente específico que inhibe la proliferación de los linfocitos T. ${ }^{15}$ Otros tratamientos de aplicación local como azatioprina, y a-crimotripsin no han tenido éxito. ${ }^{16}$ La excisión quirúrgica constituye el manejo de elección aunque no es infrecuente la recurrencia de las membranas y la necesidad de repetir el procedimiento.

Se ha informado que la combinación de excisión quirúrgica con trasplante de membrana amniótica reduce la tasa de recurrencia. ${ }^{17}$ La terapia tópica con heparina ha demostrado algunos resultados positivos cuando se usa por corta duración luego de la excisión quirúrgica ${ }^{18,19}$, sin embargo a 
largo plazo no se conocen sus resultados.La aplicación de plasminógeno o plasma fresco congelado ha mostrado ser efectiva para controlar el desarrollo de membranas ${ }^{20,21,}$ sin embargo ambos métodos tienen desventajas significativas que limitan su aplicación clínica. El plasma fresco congelado genera un riesgo potencial de reacciones inmunológicas como alergia, anafilaxis, hemólisis y lesiones pulmonares agudas relacionadas con la trasfusión. Por otra parte, el uso de plasminógeno está restringido por la distribución global limitada, accesibilidad y costo. La remoción quirúrgica de estas lesiones sin un tratamiento tópico o sistémico con productos que contengan plasminógeno, como el plasma fresco congelado o plasminógeno concentrado, es un potente determinante de la recurrencia de pseudomembranas leñosas, las cuales por lo regular reaparecen a los pocos días. ${ }^{16}$ Recientemente algunos autores reportaron el manejo tópico o sistémico exitoso con plasminógeno concentrado preparado a partir de plasma fresco congelado, pero este compuesto como solución oftalmológica con frecuencia no está comercialmente disponible.

\section{CONFLICTOS DE INTERÉS}

Los autores declaran no tener ningún conflicto de intereses.

\section{REFERENCIAS}

1. Schuster V, Seregard S. Ligneous conjunctivitis. Surv Ophthalmol. 2003;48(4):369-88. doi: https://doi.org/10.1016/ S0039-6257(03)00056-0

2. Schuster V, Hugle B, Tefs K. Plasminogen deficiency. J Thromb Haemost. 2007;5(12):2315-22. doi: https://doi.org/10.1111/ j.1538-7836.2007.02776.x

3. El-Darouti M, Zayed AA, El-Kamah GY, Mostafa MI. Ligneous conjunctivitis with oral mucous membrane involvement and decreased plasminogen level. Pediatric dermatology. 2009;26(4):448-51. doi: https://doi.org/10.1111/j.15251470.2009.00951.x

4. Tok OY, Kocaoglu FA, Tok L, Burcu A, Ornek F. Treatment of ligneous conjunctivitis with amniotic membrane transplantation and topical cyclosporine. Indian J Ophthalmol. 2012;60(6):5636. doi: https://doi.org/10.4103/0301-4738.103800

5. Tefs K, Gueorguieva M, Klammt J, Allen CM, Aktas D, Anlar FY, et al. Molecular and clinical spectrum of type I plasminogen deficiency: A series of 50 patients. Blood. 2006;108(9):3021-6. doi: https://doi.org/10.1182/blood-2006-04-017350

6. Boral M. Un nouveau syndrome palpebral. Bull Soc $\mathrm{Fr}$ Ophthalmol 1993;46:168-80.

7. Bouisson M. Ophthalmie sur-aigue avec formation de pseudomembranes a la surface de la conjoctive. Ann Ocul 1847;100:100-4.

8. Lijo Pavia J. Tumor inflamatorio fungoso recidivante de la conjuntiva palpebral. Archivos de Oftalmología HispanoAmericanos. 1925;25(289):1-11.
9. Hiremath M, Elder J, Newall F, Mitchell S, Dyas R, Monagle P. Heparin in the long-term management of ligneous conjunctivitis: a case report and review of literature. Blood Coagul Fibrinolysis. 2011;22(7):606-9. doi: https://doi.org/10.1097/ MBC.0b013e3283494b52

10. Schafer BM, Maier K, Eickhoff U, Todd RF, Kramer MD. Plasminogen activation in healing human wounds. Am J Pathol. 1994;144(6):1269-80.

11. Fuentes-Paez G, Herreras JM, del CMM, Saorni MA. Ligneous conjunctivitis in a patient with Crohn's disease. Clin Ophthalmol. 2008;2(1):203-6.

12. Hidayat AA, Riddle PJ. Ligneous conjunctivitis. A clinicopathologic study of 17 cases. Ophthalmology. 1987;94(8):949-59. doi: https:// doi.org/10.1016/s0161-6420(87)33341-x

13. Holland EJ, Chan CC, Kuwabara T, Palestine AG, Rowsey JJ, Nussenblatt RB. Immunohistologic findings and results of treatment with cyclosporine in ligneous conjunctivitis. Am J Ophthalmol. 1989;107(2):160-6. doi: https://doi. org/10.1016/0002-9394(89)90034-2

14. Klebe S, Walkow T, Hartmann C, Pleyer U. Immunohistological findings in a patient with unusual late onset manifestations of ligneous conjunctivitis. Br J Ophthalmol. 1999;83(7):878-9. doi: https://doi.org/10.1136/bjo.83.7.878a

15. Bouchard CS, Belin MW. Immunohistologic findings and results of treatment with cyclosporine in ligneous conjunctivitis. Am J Ophthalmol. 1989;108(2):210-2. doi: https://doi. org/10.1016/0002-9394(89)90034-2

16. Pergantou H, Likaki D, Fotopoulou M, Katsarou O, Xafaki P, Platokouki H. Management of ligneous conjunctivitis in a child with plasminogen deficiency. Eur J Pediatr. 2011;170(10):1333-6. doi: https://doi.org/10.1007/s00431-011-1483-9

17. Rodriguez-Ares MT, Abdulkader I, Blanco A, TourinoPeralba R, Ruiz-Ponte C, Vega A, et al. Ligneous conjunctivitis: a clinicopathological, immunohistochemical, and genetic study including the treatment of two sisters with multiorgan involvement. Virch Arch. 2007;451(4):815-21. doi: https://doi. org/10.1007/s00428-007-0481-9

18. Barabino S, Rolando M. Amniotic membrane transplantation in a case of ligneous conjunctivitis. Am J Opthalmol. 2004;137(4):7523. doi: https://doi.org/10.1016/j.ajo.2003.09.017

19. Schuster V, Zeitler P, Seregard S, Ozcelik U, Anadol D, LuchtmanJones L, et al. Homozygous and compound-heterozygous type I plasminogen deficiency is a common cause of ligneous conjunctivitis. T Thromb Haemost. 2001;85(6):1004-10.

20. Suzuki T, Ikewaki J, Iwata H, Ohashi $Y$, Ichinose A. The first two Japanese cases of severe type I congenital plasminogen deficiency with ligneous conjunctivitis: successful treatment with direct thrombin inhibitor and fresh plasma. Am J Hematol. 2009;84(6):363-5. doi: https://doi. org/10.1002/ajh.21402

21. Caputo R, Pucci N, Mori F, Secci J, Novembre E, Frosini R. Long-term efficacy of surgical removal of pseudomembranes in a child with ligneous conjunctivitis treated with plasminogen eyedrops. Thromb Haemost. 2008;100(6):1196-8. 